\title{
Current Investigations on Early Diagnosis and Treatment on Medullary Carcinoma of Thyroid Gland
}

\author{
Huma Naz ${ }^{1}$, Ayesha Nazir², Mehwish Sikander ${ }^{3}$, Zeeshan Akbar ${ }^{4}$, Sobia Yousaf ${ }^{1}$, Sidra Riaz $^{5}$ and Faiza Naseer $^{1,6 *}$ \\ ${ }^{1}$ Faculty of Pharmaceutical Sciences, Government College University, Faisalabad, Pakistan \\ ${ }^{2}$ Faculty of pharmacy and alternative medicine, Islamaia University, Bahawalpur, Pakistan
}

${ }^{3}$ College of Pharmacy, University of Sargodha, Pakistan

${ }^{4}$ Department of Pharmacy, University of Lahore, Pakistan

${ }^{5}$ Drug testing laboratories, Lahore, Pakistan

${ }^{6}$ Department of BMS, Shifa College of Pharmaceutical Sciences, Islamabad, Pakistan

Submission: March 13, 2019; Published: April 02, 2019

*Correspondence Author: Faiza Naseer, Department of BMS, Shifa College of Pharmaceutical Sciences, Islamabad, Pakistan

\begin{abstract}
Thyroid cancers are most commonly hit endocrine disease in general population. They are categorized as benign and malignant types. Medullary thyroid carcinoma (MTC) is a rare thyroid malignancy associated with a higher incidence of distant metastasis and poorer prognosis compared with the more frequently encountered well-differentiated papillary and follicular thyroid carcinomas. MTC is characterized by a sheet like growth pattern and is composed of round polygonal or spindle shaped tumor cells. Elevated serum levels of Calcitonin or CEA are indication of metastases. Cervical ultrasound is considered to be essential in the diagnosis of lymph node metastasis. CT and MRI scanning are involved in more accurate assessment of infiltration of neighboring structures. FNAB with calcitonin concentration measurement in biopsy needle washout fluid demonstrates to be supportive in diagnosis. The radiophar4maceuticals 131I or 123I-labelled metaiodobenzylguanidine (MIBG) 99mTc(V)DMSA (five value 99m Tc-labeled dimercaptosuccinic acid), 111In-pentetreotide and 99m Tc-EDDA/HYNIC- tyr3-octreotide are used in Plannar scintigraphy and single-photon emission computed tomography (SPECT). Total thyroidectomy is a surgical procedure which is performed to treat various thyroid diseases. Sunitinib, lenvatinib and vandetanib are under investigation treatment options for MTC. Total thyroidectomy provides little significant advantage of being safer procedure compared to subtotal thyroidectomy.
\end{abstract}

Keywords: Medullary thyroid carcinoma; Thyroid cancers; Thyroidectomy; Metastases

\section{Introduction}

Thyroid disorders are most commonly hit endocrine disease in general population [1]. The thyroid is a small endocrine gland which produces hormones that regulate various metabolic activities of the body [2]. Thyroid diseases can be grouped into benign and malignant types. In benign cases, the common diseases encountered are thyroiditis (mostly Hashimoto thyroiditis), goiter, thyroid adenoma, and so forth [3]. Medullary thyroid carcinoma (MTC) is a rare thyroid malignancy associated with a higher incidence of distant metastasis and poorer prognosis compared with the more frequently encountered welldifferentiated papillary and follicular thyroid carcinomas $[4,5]$.

\section{Pathology}

MTC is a type of neuroendocrine tumor which is categorized as part of apudoma ("apud" denotes to amine precursor uptake and decarboxylation. In thyroid gland, parafollicular cells derived from neural crest, are duly responsible for occurrence of medullary thyroid carcinoma. MTCs are generally well-defined tumors of variable size [6-8]. Hereditary MTCs are often bilateral and multifocal; these features are less common in sporadic MTC [9-12]. Microscopically, the classic MTC is characterized by a sheet like growth pattern and is composed of round polygonal or spindle shaped tumor cells. The nuclei are fairly uniform in shape and have coarsely clumped chromatin and inconspicuous nucleoli. The cytoplasm may have a granular appearance and can be either eosinophilic or basophilic. Necrosis and hemorrhage appear more often in larger tumors. Stromal amyloid deposits may be seen in up to $80 \%$, a unique feature not seen in other thyroid tumors [12,13]. Although the diagnosis of MTC is usually straightforward on standard histopathology, diagnostic dilemmas can occur [13]. For example, MTC may represents some features of papillary carcinoma like pseudo inclusions or psammoma bodies or follicular thyroid carcinoma by imitating follicular pattern or undifferentiated thyroid cells by signifying 
spindle cells or giant cells. In such cases, positive immunehistochemical staining for calcitonin and other neuroendocrine markers, such as chromogranin and synaptophysin, is usually diagnostic.

\section{How does medullary carcinoma present?}

Early detection of this tumor may significantly improve chances of survival [6].

\section{How should a thyroid lump be investigated?}

Elevated serum levels of Calcitonin or CEA are indication of metastases. But these tumor markers are not sufficient to start therapies. Elevated tumor markers and especially a low doubling time of serum calcitonin and CEA are rather considered risk factors for the presence and development of metastases and a poor prognosis. Those patients are being followed with serum tumor marker measurements and regular imaging, the interval depending on the serum marker level and doubling time $[5,14]$. Imaging procedures in metastatic MTC include conventional contrast-enhanced spiral CT scan or MRI of the brain, neck, chest and liver and bone scintigraphy [3]. Ultrasonography, computed tomography (CT) and Magnetic resonance imaging (MRI) are different diagnostic techniques to measure tumor invasion of surrounding muscles, trachea, larynx, esophagus. Retro-esophageal space and mediastinum. Medullary thyroid carcinoma (MTC) is insignificantly different from other thyroid carcinomas when observed in ultrasonography imaging. Parafollicular C cells are the area from where tumor originates, these areas are mainly located in the upper and central parts of the lobes. In heritable cases, lesions are basically multifocal, and may be located in both lobes [1]. In Ultrasonography, the malignancy of thyroid tumor represents as solid structure, hypo echogenicity, with improved central flow, uneven margin, its anterior-posterior dimensions greater than the transverse one and internal calcifications $[12,13]$. As compared to the papillary cancer, however, medullary thyroid carcinoma more often shows the features of benign lesions including oval shape, homogenous structure, cystic degenerative lesions or smooth well-defined margins [14]. Medullary thyroid carcinoma often does not show the characteristics for cancer [15]. As, calcitonin is considered to be a biological marker for MTC, therefore many experts used calcitonin determination in the diagnostic procedure for thyroid focal lesions. Ultrasound guided Fine needle aspiration biopsy (FNAB) is considered a standard in the diagnosis for thyroid. The sensitivity value of FNAB in the case of MTC is $44-63 \%[16,17]$. Immunocytochemical detection of concentration of calcitonin, calcitonin gene-related peptide (CGRP), neuron specific enolase (NSE) as well as calcitonin measurement in FNAB washout fluid, increase the diagnostic value of FNAB [1].

\section{Monitoring of Patients}

To monitor the recurrence of medullary thyroid carcinoma evaluating the tumor location is most essential prognostic element. After surgery, a patient is periodically monitored for serum concentration of calcitonin and CEA. The first test is usually recommended after 3 months of surgery, probably due to high level of calcitonin during wound healing and long halflife of calcitonin and CEA [1]. Depending upon the results, more measurements should be made. If the concentration of calcitonin and CEA are undetectable, then test should be suggested every 6 months in the first year and then every 12 months; but if the concentrations are detectable, at least every 6 months [1]. If calcitonin and CEA arte lying in undetectable or normal concentrations, then ultrasound of neck should be performed [18].

\section{Diagnosis of local Recurrence and Lymph Node Metastasis}

The most common site of medullary thyroid carcinoma (MTC) metastasis is lymph node. At diagnosis time, metastasis of lymph nodes ranges between $75-81 \%$ and distant ones present up to $13 \%$ [9-11]. When the concentration of calcitonin is upto $150 \mathrm{pg} / \mathrm{ml}$ then the ultrasound of neck is performed as first line medical examination to assess the metastasis or local recurrence to the neck lymph node [19]. Shape of lymph nodes with metastatic lesions are normally round or oval in shape, with obscure hilum, raised heterogeneous echogenicity, contain cystic degenerative lesions or calcification and have chaotic blood flow in Doppler imaging. These nodes are found in retrotracheal and para-and retro-esophageal space. These nodes are undetectable by ultrasound technique which sensitivity is about $46-88 \%$ but CT scan (sensitivity about $42-86 \%$ ) and MRI of neck (sensitivity about 38-91\%) are complementary techniques helpful in visualizing these nodes. [17-20].

FNAB plays a vital role in the diagnosis of nodal metastasis, especially in the case when small nodes were found without significant morphological change. Calcitonin concentration can also be assessed in biopsy needle wash-out fluid in FNAB [18]. Local recurrence is often difficult to detect because of small size and problem associated with differentiating it from scar changes in tumor. Cervical ultrasound is considered to be essential in the diagnosis of lymph node metastasis. CT and MRI scanning are involved in more accurate assessment of infiltration of neighboring structures. FNAB with calcitonin concentration measurement in biopsy needle washout fluid demonstrates to be supportive in diagnosis [18].

\section{Diagnosis of Distant Metastasis}

When concentration of calcitonin exceeds $150 \mathrm{pg} / \mathrm{ml}$ then computed tomography, magnetic resonance imaging or positron emission tomography are recommended to diagnose distant metastasis. if calcitonin value is less than $150 \mathrm{pg} / \mathrm{ml}$, then these methods cannot detect tumor foci. Diagnostic tests for distant metastasis, including computed tomography, magnetic resonance imaging or positron emission tomography are only recommended when concentration of calcium exceeds $150 \mathrm{pg} /$ $\mathrm{ml}$, because below this value, tumor foci cannot be detected 


\section{Cancer Therapy \& Oncology International Journal}

by these methods $[1,19]$. Another important point should be remembered that at high concentration of up to $1000 \mathrm{pg} / \mathrm{ml}$, risk of false negative imaging results remain high [19]. Most probable cause of false negative imaging are micro-metastasis to the liver, which can be detectable by laparoscopy [20]. Medullary thyroid carcinoma (MTC) is one of the cancer in which liver metastasis are hyper echogenic on ultrasound [21]. Occasionally, in the ultrasound low or mixed echogenicity is possessed by metastasis due to the presence of fluid and calcification. In such cases CT and MRI are recommended tests to make a difference from haemagioma $[11,21,22]$. Their vascularization is very well developed. The most sensitive diagnostic imaging technique for MTC metastasis to liver is dynamic MRI [16].

Medullary thyroid carcinoma metastasis in lungs may be micro or macro-nodular [11]. These are small and in the form of multiple lesions in majority of cases $[1,11]$. Sometimes these lesions appear in the form of miliary spread, perihilar fibro-nodular lesions or calcified masses [23,24]. CT scan provides maximum sensitivity in detection of metastasis to lungs and mediastinum $[1,16]$. Metastasis of MTC to bones are predominantly osteolytic and less often osteoblastic, however both forms of metastasis coexist [25]. Classis bone scinitigraphy with hydroxyl methylene diphosphonate or $99 \mathrm{~m}$ Tc labeled methylene diphosphonate are responsible to detect osteoblastic lesions which are highly prone to capturing radioisotopes, in the case of medullary thyroid carcinoma provides the sensitivity of about 58-73\% [16,26]. MRI technique is responsible to provide higher sensitivity in the detection of MTC metastasis approximately about $61-100 \% \quad[16,26]$. In T1-weighted sequence, metastasis is less intensive, but may be enhanced after administration of gadolinium. MRI technique has more sensitivity in detection of lesions in the axial skeleton, while classic scintigraphy considered to be more sensitive in detection of lesions in long bones metastasis [16]. In diagnostic measures of MTC metastasis toward bones, both MRI and scintography are equally complementary so when they are used in combination the sensitivity can be boosted up to $61-94 \%$ [16].

\section{Nuclear Medicine Imaging in the diagnosis of Medullary Thyroid Carcinoma (MTC)}

Plannar scintigraphy and single-photon emission computed tomography (SPECT). The radiopharmaceuticals emitting $\gamma$ radiation used for Medullary Thyroid Carcinoma (MTC) scintigraphic diagnosis include:

131I or 123I-labelled metaiodobenzylguanidine (MIBG) $99 \mathrm{mTc}(\mathrm{V})$-DMSA (five value $99 \mathrm{~m}$ Tc-labeled dimercaptosuccinic acid), 111In-pentetreotide and 99m Tc-EDDA/HYNIC- tyr3octreotide. MIBG is a noradrenalin analog specific for tumors developing from neural crest [27]. Primarily used in the diagnosis of neuroblastoma and Pheochromocytoma, it may also be useful for MTC foci imaging [27]. The available preprations are 131I and 123I-labelled, and due to enhanced dosimetric characteristics of 123I, the scintigraphy by the use of 123I-MIBI results in improved imaging sensitivity enhanced quality [28]. On contrary, 131I-MIBI due to $\alpha$ radiation may be used in the case of palliative treatment of MTC, although this treatment option often considered as ineffective [1,29]. In the case of MTC, the Specificity of MIBG scintigraphy is greater up to $95 \%$, but its sensitivity is fewer about $25-30 \%$ only, and majorly it is based on the level \& degree of differentiation of the tumor. It is larger in patients with MEN 2A syndrome than in sporadic Medullary Thyroid Carcinoma [30,31]. Greater sensitivity of MIBG scintigraphy was observed in the case of localization of persistent tumor cells and localized MTC recurrence, as compared with distant metastasis, which is explained by progressive differentiation of metastatic lesions $[28,30]$.

Scintigraphy along with $99 \mathrm{~m}$ Tc(V)-DMSA can be used both in the diagnosis of the primary foci and the recurrence of MTC $[31,32]$. Its sensitivity value fluctuates in different studies is about $50-80 \%[31,33]$. Depending on the type of tissue, $99 \mathrm{mTc}(\mathrm{V})$-DMSA uptake is significantly high in soft tissues as compare to bone [28]. Somatostatin receptor scintigraphy with the use of $111 \mathrm{In}$-pentetreotide or 99m Tc-EDDA/ HYNICTyr3-octreotide is also a helpful tool for MTC diagnosis [33-40]. Along with imaging of tumor foci, it is essential in the selection of patients with unrespectable MTC to be treated with hot and cold Somatostatin analogues $[35,36]$. There is great variation in the results of the evaluation of octreScan sensitivity in the detection of MTC ranges from 20 to $78.5 \%$ [27,28,33]. According to numerous studies, this value is measured to be lesser than that of the conventional imaging including X-rays, ultrasound, bone scintigraphy or CT scan [34]. The studies of comparison of $111 \mathrm{In}$-pentetreotide scintigraphy with $99 \mathrm{mTc}(\mathrm{V})$-DMSA and MIBG, defines that its sensitivity is greater than that of MIBG and comparable to or more than $99 \mathrm{mTc}(\mathrm{V})$-DMSA $[33,35]$.

In well differentiated tumors the uptake of 111Inpentetreotide is higher, but observed significantly less in tumors undergo rapid progression and distant metastasis, that is because of decreased expression of Somatostatin receptors in less differentiated tumors [36]. The sensitivity of $99 \mathrm{~m}$ TcEDDA/HYNIC-Tyr3-octreotide scintigraphy, compared to 111 In-pentetreotide is greater $[37,38]$. This value is evaluated as $56.2-79.5 \%[39,40]$. It has been found that results of $99 \mathrm{mTc}-$ EDDA/HYNIC Tyr3-octreotide scintigraphy were little worse than 18F-FDG PET, while better than 99mTc-MIBI scintigraphy [40]. By using scintigraphy, good results were obtained based upon monoclonal antibodies against carcino-embryonic antigen (CEA) labeled with isotopes 99m Tc, 123I, 131I and 111In [36]. The anti-CEA antibodies scintigraphy indicates sensitivity 50$78 \%$, somewhat less or comparable with 99m Tc (V)-DMSA, and higher than 131I-MIBG $[36,41,42]$. Contrary to Somatostatin receptor scintigraphy, this diagnostic technique proved to be beneficial for extremely aggressive MTC [36]. 131I-labeled antiCEA antibodies show effectiveness in palliative treatment [36]. The effectiveness of $201 \mathrm{Tl}, 99 \mathrm{mTc}-\mathrm{MIBI}$ and $111 \mathrm{In}$-labeled 


\section{Cancer Therapy \& Oncology International Journal}

calcitonin antibodies scintigraphy was also evaluated, but the studies evaluating those methods in the diagnostic procedures of MTC indicate, that these methods have less sensitivity as compared to other procedures $[40,41,43]$.

\section{Positron Emission Tomography (PET)}

18F-fluorodeoxyglucose(18F-FDG) was 1st radiopharmaceutical emitting $\beta+$, that is used for neoplasm as diagnostic radiopharmaceutical by positron emission tomography [44]. Studies have shown in less differentiated and rapid growing tumors there is more uptake of $18 \mathrm{~F}-\mathrm{FDG}$, which rapidly metabolize glucose [44]. Sensitivity of 18F-FDG PET ranges from 17 to $95 \%$ in diagnosis of recurrence of MTC [4549]. It has been found that it is greater in patients having larger tumors (calcitonin is greater than $1000 \mathrm{pg} / \mathrm{ml}$ ), as well as in patients with quickly proliferating (with calcitonin concentration doubling time less than 2 years) and less differentiated tumors $[16,45,50]$.

In patients of MTC, Positive result of 18F-FDG PET is related to worse prognosis. Patients having calcitonin level less than $1000 \mathrm{pg} / \mathrm{ml}$ has a limited use of 18F-FDG PET, as in that situation the range of sensitivity is from $20-37 \%[16,50]$. In patients with MEN 2A, Sensitivity of 18F-FDG PET is significantly less due to less aggressive course of MTC in such patients [50]. Major advantage of 18-FDG PET is entire body imaging capability as compare to conventional diagnostic imaging. Although this is not considered to be a radiopharmaceutical of choice in well differential tumors, including MTC, it has been found that 18-FDG PET has more sensitivity as compare to SPECT $[40,44,47]$. The sensitivity of 18F-FDG PET depends on the size and location of lesions $[16,46]$. Studies showed that this is valuable in detection of the neck and mediastinum lymph node metastasis and local recurrence, defines more sensitivity compared to MRI or CT scan $[16,46,47]$. In detection of small metastasis which are located in the liver and lungs, 18F-FDG PET is less sensitive as compare with CT scan, and worse sensitive than MRI or scintigraphy in the bone metastasis imaging $[16,46,47]$.

Another radiopharmaceutical, 18F-DOPA, is effectively used in MTC PET collected by the cells liable for decarboxylation and up taking catecholamine precursors [48]. 18F-FDG PET estimated about $47-83 \%$ is less than the sensitivity of 18F-DOPA PET [17,25,48-52]. These two diagnostic examinations are considered to be complement for each other, as 18F-DOPA can be used for the detection of MTC having less proliferation capacity, and 18F-FDG can be used for more aggressive tumors with doubling time of calcitonin less than 12 months [25]. 18F-DOPA PET/CT is specifically useful in detection metastatic lymph nodes and also allow to detect those tumor foci which are at calcitonin concentration greater than $150 \mathrm{pg} / \mathrm{ml}$ [52]. In the PET diagnosis of MTC, 68Ga-labeled Somatostatin analogs (68Ga-DOTA-NOC, 68Ga-DOTA-TOC, 68Ga-DOTA-TATE) all are important radiopharmaceuticals [49,53,54]. In detection of MTC recurrences, sensitivity of $68 \mathrm{Ga}$-DOTA-TATE PET/CT is $72 \%$ compare to 18F-FDG PET/CT which is 78\% [53]. By means of $\mathrm{PET} / \mathrm{CT}$, evaluation of somatostatin receptor expression can be helpful in selection of patients for treatment with "hot" (177Luor 90Y-DOTA-TATE) or "cold" (pasireotide) somatostatin analogues [54]. After comparing the results of PET/CT in those patients having persistent and recurrent MTC, it has been found that 18F-DOPA has more sensitivity for cancer foci detection when compared with 18F-FDG and Somatostatin analogues[49]. Expansiveness and least availability are disadvantages of this method, as well 68Ga-DOTA-TATE PET/CT and 18F-FDG PET/CT found to have no significant differences in sensitivity in result evaluation of these two diagnostic examinations $[49,53]$

\section{How is the diagnosis of medullary carcinoma confirmed?}

Imaging plays a critical role in both early detection and accurate staging of MTC, which dictates surgical management. Furthermore, MTC may recur even after radical surgical resection, and imaging plays a crucial role in follow-up to detect early recurrence. In addition, $25 \%$ of MTCs occur as hereditary forms, the majority of which occur as part of the multiple endocrine neoplasia (MEN) 2 syndromes $[7,8]$

\section{What needs to be discussed before thyroidectomy?}

Treatment goals in advanced MTC are ultimately related to promoting the quality of life. It is important to explicitly discuss with the patient the expectations and goals with respect to quality of life in the individual context and to agree on a personalized treatment plan. In general, it is important to document the sites of metastases and the tumor volume as well as tumor progression to get an estimate of total tumor burden and prognosis. In addition, it is necessary to document signs and symptoms and estimate imminent problems. A satisfactory quality of life can usually be maintained, in patient with recurrent disease, for months or even years. Treatment choices must stabilize the typically slow progression rate and the rational life expectancy against the efficacy and side effects of treatments [52-54]. A vital step is to choose whether any therapy be palliative or curative, because the risk-benefit ratio of palliative and curative treatments is different. Curative treatments are defined as improved as well as disease-free survival. The main objective of palliative therapies is to improve quality of life by relief of symptoms. When recurrent local disease is present or distant metastases are limited to a single organ curative surgical resection may be considered. Unfortunately, in advanced MTC, curative options are limited as both local recurrent disease and metastases are seldom amenable to curative surgery. Brain metastases, airway obstruction, threatening spinal cord compression, impending, bleeding or active fracture or hormonal secretion are critical locations for lesions and require an active treatment $[9,54]$.

\section{Treatment under Investigation}

i. Sunitinib showed remarkable decrease in calcitonin levels in MTC patients but under investigation due to some cardiac toxicities $[55,56]$. 


\section{Cancer Therapy \& Oncology International Journal}

ii. Lenvatinib showed promising antitumor activity in various human thyroid cancer xenograft models derived from DTC, MTC, and ATC cell lines [57].

iii. Vandetanib has shown efficacy in patients with locally advanced or metastatic MTC $[58,59]$.

\section{Treatment}

Total thyroidectomy is a surgical procedure which is performed to treat various thyroid diseases wherein the thyroid gland is removed. But the use of total thyroidectomy procedure is considered not to be safe for thyroid carcinomas and also for treatment of few benign diseases because of the risks involved [53]. Total thyroidectomy is a safe and effective procedure for most of the thyroid diseases in the hands of an expert surgeon. Subtotal thyroidectomy is similarly effective, but it is associated with significant recurrence rate and may leave few traces of inadequately treated thyroid cancers. Thus, total thyroidectomy provides little significant advantage of being safer procedure compared to subtotal thyroidectomy.

\section{Concluding Remarks}

To cope with different thyroid cancers especially medullary thyroid carcinoma proper diagnosis in early stages helps in the treatment and better prognosis of disease.

\section{References}

1. Wells SA Jr, Asa SL, Dralle H, Elisei R, Evans DB, et al. (2015) Revised American Thyroid Association guidelines for the management of medullary thyroid carcinoma. Thyroid 25(6): 567-610.

2. Wolinski K, Rewaj-Losyk M, Ruchala M (2014) Sonographic features of medullary thyroid carcinomas -a systematic review and meta-analysis. Endokrynol Pol 65(4): 314-318.

3. Choi N, Moon WJ, Lee JH, Baek JH, Kim DW, et al. (2011) Ultrasonographic findings of medullary thyroid cancer: differences according to tumour size and correlation with fine needle aspiration results. . Acta Radiol 52(3): 312-316.

4. Trimboli P, Giovanella L, Valabrega S, Andrioli M, Baldelli R, et al (2014) Ultrasound features of medullary thyroid carcinoma correlate with cancer aggressiveness: a retrospective multicenter study. . J Exp Clin Cancer Res 33(1): 87.

5. Andrioli M, Trimboli P, Amendola S, Valabrega S, Fukunari N, et al. (2014) Elastographic presentation of medullary thyroid carcinoma. Endocrine 45(1): 153-155.

6. Essig GF Jr, Porter K, Schneider D, Debora A, Lindsey SC, et al. (2013) Fine needle aspiration and medullary thyroid carcinoma: the risk of inadequate preoperative evaluation and initial surgery when relying upon FNAB cytology alone. Endocr Pract 19(6): 920-927.

7. Bugalho MJ, Santos JR, Sobrinho L (2005) Preoperative diagnosis of medullary thyroid carcinoma: fine needle aspiration cytology as compared with serum calcitonin measurement. J Surg Oncol 91(1): 56-60.

8. Jarząb B, SS, Lange D (2010) Diagnostyka i leczenie raka tarczycy- rekomendacje polskie. Endokrynol Pol 61(5): 518-568.

9. Moley JF, DeBenedetti MK (1999) Patterns of nodal metastases in palpable medullary thyroid carcinoma. recommendations for extent of node dissection. Ann Surg 229(6): 880-887.

10. Weber T, Schilling T, Frank-Raue K, Colombo-Benkmann M, Hinz U, et al. (2001) Impact of modified radical neck dissection on biochemical cure in medullary thyroid carcinomas. Surgery 130(6): 1044-1049.

11. Ganeshan D, Paulson E, Duran C, Cabanillas ME, Busaidy NL, et al. (2013) Current update on medullary thyroid carcinoma. AJR Am Roentgenol 201(6): W867-W876.

12. Raue F, Winter J, Frank-Raue K, Lorenz D, Herfarth C, et al. (1989) Diagnostic procedure before reoperation in patients with medullary thyroid carcinoma Horm Metab Res Suppl 21: 31-34.

13. Dörr U, Würstlin S, Frank-Raue K, Raue F, Hehrmann R, et al. (1993) Somatostatin receptor scintigraphy and magnetic resonance imaging in recurrent medullary thyroid carcinoma: a comparative study. Horm Metab Res Suppl 27: 48-55.

14. Wang Q, Takashima S, Fukuda H, Takayama F, Kobayashi S, et al. (1999) Detection of medullary thyroid carcinoma and regional lymph node metastases by magnetic resonance imaging. Arch Otolaryngol Head Neck Surg 125: 842-848.

15. Kebebew E, Kikuchi S, KS Duh QY, Clark OH (2000) Long-term results of reoperation and localizing studies in patients with persistent or recurrent medullary thyroid cancer. Arch Surg 135(8): 895-901.

16. Giraudet AL, Vanel D, Leboulleux S, Aupérin A, Dromain C, et al. (2007) Imaging medullary thyroid carcinoma with persistent elevated calcitonin levels. J Clin Endocrinol Metab 92(11): 4185-4190.

17. Sesti A, Mayerhoefer M, Weber M, Anner P, Wadsak W, et al. (2014) Relevance of calcitonin cut-off in the follow-up of medullary thyroid carcinoma for conventional imaging and 18-fluorine-fluorodihydroxyphenylalanine PET. Anticancer Res 34(11): 6647-6654.

18. Boi F, Maurelli I, Pinna G, Atzeni F, Piga M, et al. (2007) Calcitonin measurement in wash-out fluid from fine needle aspiration of neck masses in patients with primary and metastatic medullary thyroid carcinoma. J Clin Endocrinol Metab 92(6): 2115-2118.

19. Jarząb B, SS, Lange D (2010) Diagnostyka i leczenie raka tarczycy- rekomendacje polskie. Endokrynol Pol 61(5): 518-568.

20. Tung WS, Vesely TM, Moley JF (1995) Laparoscopic detection of hepatic metastases in patients with residual or recurrent medullary thyroid cancer. Surgery 118(6): 1024-1029.

21. Leclère J, Sidibé S, Lassau N, Gardet P, Caillou B, et al., Ultrasonographic aspects of hepatic metastases of thyroid medullary cancers. J Radiol 77(2): 99-103.

22. Van Beers B, PJ, Defalque D (1990) Hepatic metastases in medullary thyroid carcinoma: possible pitfall with MR imaging. Eur J Radiol 11(2): 107-109.

23. Hung WW, Wang CS, Tsai KB, Ou-Yang F, Shin SJ, et al. (2009) Medullary thyroid carcinoma with poor differentiation and atypical radiographic pattern of metastasis. Pathol Int 59(9): 660-663.

24. Mazoyer G, Cordier JF, Zabern JM, Vergnon JM, Berthezene F, et al (1986) Pulmonary metastases in medullary cancers of the thyroid. Study of 4 cases. Originality of the lymphangitic form with amyloid stroma. . Rev Mal Respir 3(3): 139-143.

25. Treglia G, Villani MF, Giordano A, Rufini V (2012) Detection rate of recurrent medullary thyroid carcinoma using fluorine-18 fluorodeoxyglucose positron emission tomography: a meta-analysis. Endocrine, 42(3): 535-545.

26. Mirallié E, Vuillez JP, Bardet S, Frampas E, Dupas B, et al. (2005) High frequency of bone/bone marrow involvement in advanced medullary thyroid cancer. J Clin Endocrinol Metab 90(2): 779-788.

27. Rufini V, Castaldi P, Treglia G, Perotti G, Gross MD, et al. (2008) Nuclear medicine procedures in the diagnosis and therapy of medullary thyroid carcinoma. Biomed Pharmacother 62(3): 139-146.

28. Skoura E (2013) Depicting medullary thyroid cancer recurrence: the past and the future of nuclear medicine imaging. Int J Endocrinol Metab 11(4): e8156. 


\section{Cancer Therapy \& Oncology International Journal}

29. Maiza JC, Grunenwald S, Otal P, Vezzosi D, Bennet A, et al. (2012) Use of 131I-MIBG therapy in MIBG-positive metastatic medullary thyroid carcinoma. Thyroid 22(6): 654-655.

30. Baulieu JL, Guilloteau D, Delisle MJ, Perdrisot R, Gardet P, et al. (1987) Radioiodinated meta-iodobenzylguanidine uptake in medullary thyroid cancer. A French cooperative study. Cancer 60(9): 2189-2194

31. Baulieu JL, Guilloteau D, Delisle MJ, Perdrisot R, Gardet P, et al. (1989) The role of radiopharmaceuticals MIBG and (V) DMSA in the diagnosis of medullary thyroid carcinoma. Henry Ford Hosp Med J 37(3-4): 175177.

32. Clarke S, Lazarus C, Maisey M (1989) Experience in imaging medullary thyroid carcinoma using 99mTc (V)-dimercaptosuccinic acid (DMSA) Henry Ford Hosp Med J 37(3-4): 167-168.

33. Arslan N, Ilgan S, Yuksel D, Serdengecti M, Bulakbasi N, et al. (2001) Comparison of In-111 octreotide and Tc-99m (V) DMSA scintigraphy in the detection of medullary thyroid tumor foci in patients with elevated levels of tumor markers after surgery. Clin Nucl Med 26(8): 683-688.

34. Baudin E, Lumbroso J, Schlumberger M, Leclere J, Giammarile F, et al. (1996) Comparison of octreotide scintigraphy and conventional imaging in medullary thyroid carcinoma. J Nucl Med 37(6): 912-916.

35. Kaltsas G, Korbonits M, Heintz E, Mukherjee JJ, Jenkins PJ, et al. (2001) Comparison of somatostatin analog and meta-iodobenzylguanidine radionuclides in the diagnosis and localization of advanced neuroendocrine tumors. J Clin Endocrinol Metab 86(2): 895-902.

36. Behr TM, Gratz S, Markus PM, Dunn RM, Hüfner M, et al. (1997) Anti-carcinoembryonic antigen antibodies versus somatostatin analogs in the detection of metastatic medullary thyroid carcinoma: are carcinoembryonic antigen and somatostatin receptor expression prognostic factors? Cancer 80: 2436-2457.

37. Decristoforo C, Mather SJ, Cholewinski W, Donnemiller E, Riccabona G, et al. (2000) 99mTc-EDDA/HYNIC-TOC: a new 99mTc-labelled radiopharmaceutical for imaging somatostatin receptor-positive tumours; first clinical results and intra-patient comparison with 111In-labelled octreotide derivatives. Eur J Nucl Med 27(9): 1318-1325.

38. Parisella M, D’Alessandria C, van de Bossche B, Chianelli M, Ronga G, et al. (2004) 99mTc-EDDA/ HYNIC-TOC in the management of medullary thyroid carcinoma. Cancer Biother Radiopharm 19(2): 211-217.

39. Czepczyński R, Parisella MG, Kosowicz J, Mikołajczak R, Ziemnicka K et al. (2007) Somatostatin receptor scintigraphy using 99mTc-EDDA/ HYNIC-TOC in patients with medullary thyroid carcinoma. . Eur J Nucl Med Mol Imaging 34(10): 1635-1645.

40. Sager S, Kabasakal L, Ocak M, Maecke H, Uslu L, et al. (2013) Clinical value of technetium-99m--labeled octreotide scintigraphy in local recurrent or metastatic medullary thyroid cancers: a comparison of lesions with 18F-FDG-PET and MIBI images. Nucl Med Commun 34(12): 1190-1195.

41. Sandrock D, Blossey HC, Steinroeder M, Munz DL (1989) Contribution of different scintigraphic techniques to the management of medullary thyroid carcinoma. Henry Ford Hosp Med J 37(3-4): 173-174.

42. Troncone L, Rufini V, De Rosa G, Testa A (1989) Diagnostic and therapeutic potential of new radiopharmaceutical agents in medullary thyroid carcinoma. Henry Ford Hosp Med J 37(3-4): 178-184.

43. Skoura E (2013) Depicting medullary thyroid cancer recurrence: the past and the future of nuclear medicine imaging. Int J Endocrinol Metab 11(4): e8156.

44. Ambrosini V, Tomassetti P, Franchi R, Fanti S (2010) Imaging of NETs with PET radiopharmaceuticals. Q J Nucl Med Mol Imaging 54(1): 1623.
45. Giorgio Treglia, Vittoria Rufini, Massimo Salvatori, Alessandro Giordano, et al. (2012) PET Imaging in Recurrent Medullary Thyroid Carcinoma. Int J Mol Imaging 2012: 324686.

46. Szakáll S Jr, Esik O, Bajzik G, Repa I, Dabasi G, et al. (2002) 18F-FDG PET detection of lymph node metastases in medullary thyroid carcinoma. J Nucl Med 43(1): 66-71.

47. Rubello D, Rampin L, Nanni C, Banti E, Ferdeghini M, et al. (2008) The role of 18F-FDG PET/CT in detecting metastatic deposits of recurrent medullary thyroid carcinoma: a prospective study. Eur J Surg Oncol 34(5): 581-586.

48. Beuthien-Baumann B, Strumpf A, Zessin J, Bredow J, Kotzerke J, et al. (2007) Diagnostic impact of PET with 18F-FDG, 18F-DOPA and 3-0-methyl-6-[18F]fluoro-DOPA in recurrent or metastatic medullary thyroid carcinoma. . Eur J Nucl Med Mol Imaging 34(10): 1604-1609.

49. Treglia G, Castaldi P, Villani MF, Perotti G, de Waure C, et al. (2012) Comparison of 18F-DOPA, 18F-FDG and 68Ga-somatostatin analogue $\mathrm{PET} / \mathrm{CT}$ in patients with recurrent medullary thyroid carcinoma. . Eur J Nucl Med Mol Imaging 39(4): 569-580.

50. Skoura E, Rondogianni P, Alevizaki M, Tzanela M, Tsagarakis S, et al. (2010) Role of [(18)F]FDG-PET/CT in the detection of occult recurrent medullary thyroid cancer. Nucl Med Commun 31(6): 567-575.

51. Marzola MC, Pelizzo MR, Ferdeghini M, Toniato A, Massaro A, et al. (2010) Dual PET/CT with 18F-DOPA and 18F-FDG in metastatic medullary thyroid carcinoma and rapidly increasing calcitonin levels: comparison with conventional imaging. Eur J Surg Oncol 36(4): 414-421.

52. Luster M, Karges W, Zeich K, Pauls S, Verburg FA, et al. (2010) Clinical value of 18-fluorine-fluorodihydroxyphenylalanine positron emission tomography/computed tomography in the follow-up of medullary thyroid carcinoma. Thyroid 20(5): 527-533.

53. Conry BG, Papathanasiou ND, Prakash V, Kayani I, Caplin M, et al. (2010) Comparison of 68Ga-DOTATATE and (18)F-fluorodeoxyglucose $\mathrm{PET} / \mathrm{CT}$ in the detection of recurrent medullary thyroid carcinoma. Eur J Nucl Med Mol Imaging 37(1): 49-57.

54. Verburg FA, Anlauf M, Mottaghy FM, Karges W (2015) Somatostatin receptor imaging-guided pasireotide therapy in medullary thyroid cancer with ectopic adrenocorticotropin production. Clin Nucl Med 40(1): e83-e84.

55. Ravaud A, De La Fouchardiere C, Asselineau J, Delord JP, Christine Do Cao, et al. (2010) Efficacy of sunitinib in advanced medullary thyroid carcinoma: intermediate results of Phase II THYSU. The oncologist 15(2): 212-213.

56. Ferrari SM, Centanni M, Virili C, Miccoli M, FerrariPaola P, et al. (2017) Sunitinib in the treatment of thyroid cancer. Curr Med Chem.

57. Schlumberger M, Jarzab B, Cabanillas ME, Robinson B, Pacini F, et al. A Phase II Trial of the Multitargeted Tyrosine Kinase Inhibitor Lenvatinib (E7080) in Advanced Medullary Thyroid Cancer. Clin Cancer Res 22(1): 44-53.

58. Trimboli P, Castellana M, Virili C, Giorgino F, Giovanella L, et al. (2018) Efficacy of Vandetanib in Treating Locally Advanced or Metastatic Medullary Thyroid Carcinoma According to RECIST Criteria: A Systematic Review and Meta-Analysis. Front Endocrinol (Lausanne) 9: 224.

59. Wells SA Jr, Robinson BG, Gagel RF, Dralle H, Fagin JA, et al. (2012) Vandetanib in patients with locally advanced or metastatic medullary thyroid cancer: a randomized, double-blind phase III trial. J Clin Oncol Jan 30(2): 134-141. 
This work is licensed under Creative Commons Attribution 4.0 License DOI: 10.19080/CTOIJ.2019.13.555861
Your next submission with Juniper Publishers will reach you the below assets

- Quality Editorial service

- Swift Peer Review

- Reprints availability

- E-prints Service

- Manuscript Podcast for convenient understanding

- Global attainment for your research

- Manuscript accessibility in different formats ( Pdf, E-pub, Full Text, Audio)

- Unceasing customer service

Track the below URL for one-step submission https://juniperpublishers.com/online-submission.php 